\title{
无碱参与的铜催化芳基硼酸和硫酚的偶联反应研究
}

\author{
杨明华* 裴 吉严国兵翁秋月 \\ (丽水学院理学院 丽水 323000)
}

\begin{abstract}
摘要 无碱参与的条件下, 研究了 $\mathrm{Cu}$ 盐催化芳基硼酸与硫酚的偶联反应. 通过反应溶剂、温度、铜盐、配体等条件的 优化, 结果表明在空气中, 以 1,10 -菲啰啉-醋酸铜为催化剂, $\mathrm{DMSO} / \mathrm{H}_{2} \mathrm{O}(V: V=1: 1)$ 作为溶剂, 反应温度为 $60{ }^{\circ} \mathrm{C}$, 以 较高产率合成二芳基硫醚.
\end{abstract}

关键词 偶联反应; 硫酚; 芳基硼酸; 1,10-菲啰啉; 醋酸铜

\section{Base-Free Cu(II)-Catalyzed Coupling Reaction of Arylboronic Acids and Thiols}

\author{
Yang, Minghua* Pei, Ji Yan, Guobing Weng, Qiuyue \\ (Colllege of Science, Lishui Universtiy, Lishui 323000)
}

\begin{abstract}
Baes-free $\mathrm{Cu}(\mathrm{II})$-catalyzed coupling reaction of arylboronic acid and thiols was described. After optimization of reaction conditions, such as solvents, reaction temperatures, copper salts and ligands, the results show that some biarylsulfides were synthesized in good yields using 1,10-phenanthroline- $\mathrm{Cu}(\mathrm{OAc})_{2} \cdot \mathrm{H}_{2} \mathrm{O}$ as catalyst, $\mathrm{DMSO} / \mathrm{H}_{2} \mathrm{O}(V: V=1: 1)$ as solvent, under air atmosphere at $60{ }^{\circ} \mathrm{C}$.

Keywords coupling reaction; thiol; arylboronic acid; 1,10-phenanthroline; cupric acetate
\end{abstract}

芳基硫醚是重要的有机合成中间体，广泛用于药物 化学和材料化学 ${ }^{[1]} \cdot \mathrm{Pd}^{[2]}, \mathrm{Ni}^{[3]}$ 和 $\mathrm{Cu}^{[4]}$ 等过渡金属催化合 成芳基硫醚有较多的报道 ${ }^{[5]}$, 其中最为常见的方法是 $\mathrm{Cu}$ 催化芳基卤化物与含硫的试剂，如 $\mathrm{S}^{[6]}, \mathrm{KSCN}^{[7]}$, 硫 尿 $^{[8]}$, 硫酚 ${ }^{[9]}$ ，二硫醚 ${ }^{[10]}$ 等反应合成对称或非对称的硫 醚. 芳基苯硼酸作为一种重要的芳基化试剂广泛应用于 偶联反应，但应用于硫醚的合成仅有几例报道. 2000 年 Guy 等 ${ }^{[11]}$ 研究首例在氩气中, 芳基硼酸和环己基硫醇的 $\mathrm{C}-\mathrm{S}$ 交叉偶联反应合成硫醚，反应不仅要使用 1.5 equiv.醋酸铜, 而且还要加入分子篮. 2009 年 Punniyamurthy 小组 ${ }^{[12]}$ 用纳米 $\mathrm{CuO}$ 催化了苯硼酸和苯硫酚的反 应，在 $\mathrm{N}_{2}$ 下 $80{ }^{\circ} \mathrm{C}$ 反应 $10 \mathrm{~h}$ ，仅有 $16 \%$ 的产率. 也有人用 芳基硼酸分别与 $N$-硫取代的丁二酰亚胺 ${ }^{[13]}$ 和二硫醚 ${ }^{[14]}$ 反应合成硫醚, 得到了好的结果. 就在我们准备本文时, Feng 等 ${ }^{[15]}$ 报道了在室温和氧气存在下, 加入 $0.5 \mathrm{~mL}$ 的 $40 \% n-\mathrm{BuOH}$ 为碱 $\left(0.5 \mathrm{mmol}\right.$ 底物), 用 $\mathrm{CuSO}_{4}-1,10$-菲啰 啉催化芳基嗍酸和硫酚的反应，以中等到高的收率得到
了硫醚. 我们 ${ }^{[16]}$ 的研究兴趣是催化合成 $\mathrm{C}$ 一杂键, 在重 复 Punniyamurthy 小组 ${ }^{[12]}$ 的苯硼酸和苯硫酚反应过程中, 发现反应体系中加少量水, 可以大大提高反应收率. 因 此我们对该反应作了进一步探讨, 结果表明, 不需要碱 参与, 以 $\mathrm{DMSO} / \mathrm{H}_{2} \mathrm{O}(V: V=1: 1)$ 为溶剂, 在 $\mathrm{Cu}(\mathrm{OAc})_{2}-1,10$ - 菲啰啉 $\left(1,10\right.$-phen $\left.\cdot \mathrm{H}_{2} \mathrm{O}\right)$ 催化下, 空气中 $60{ }^{\circ} \mathrm{C}$ 反应 $8 \mathrm{~h}$, 芳基硼酸和硫酚反应，可以较高产率得 到二芳基硫醚(Eq. 1).

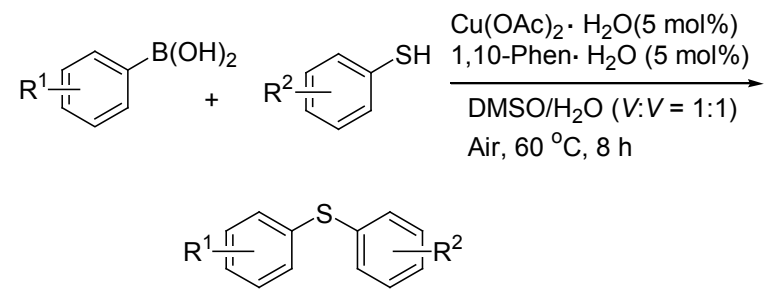

\footnotetext{
* E-mail: mhyang@1su.edu.cn

Received September 6, 2012; revised October 17, 2012; published online October 25, 2012.

Project supported by the Natural Science Foundation of Zhejiang Province (No. Y407081).

浙江省自然科学基金(No. Y407081)资助项目.
} 


\section{1 实验部分}

\section{1 仪器与试剂}

核磁共振仪: Bruker AMX-300, 以 TMS 为内标, 気代氯仿为溶剂; 部分产品的收率和表征用 GC-MS: Varian GC/MS (431 GC-220 MS). 所用药品均为市售分 析纯，除特别注明外，未经进一步处理.

\section{2 优化实验的一般步骤}

将配体 $(0.05 \mathrm{mmol})$ 、铜盐或铜氧化物 $(0.05 \mathrm{mmol})$ 和溶剂 $1.5 \mathrm{~mL}$, 分别加入反应管中, 室温下搅拌 $10 \mathrm{~min}$, 再加入苯硼酸 $(0.5 \mathrm{mmol}$ )和 3 -甲氧基苯硫酚 $(0.6 \mathrm{mmol})$, 控温 $45{ }^{\circ} \mathrm{C}$ 搅拌反应 $8 \mathrm{~h}$. 冷却至室温, 加入 $5 \mathrm{~mL}$ 乙酸乙 酯和 $3 \mathrm{~mL}$ 水, 再加入 $0.5 \mathrm{~mL}$ 浓度为 $1.0 \mathrm{~mol} / \mathrm{L}$ 二苯硫醚 的乙酸乙酯溶液, 充分搅拌后, 用 GC-MS 检测确定产 率. GC 色谱柱为 Varian VF-5ms (CP8944), $60{ }^{\circ} \mathrm{C}$ 开始以 $20{ }^{\circ} \mathrm{C} / \mathrm{min}$ 梯度升温至最高温度 $280{ }^{\circ} \mathrm{C}$, 载气流速为 0.5 $\mathrm{mL} / \mathrm{min}$; 保留时间: 内标二苯二硫醚 $8.642 \mathrm{~min}$, 产物 $9.993 \mathrm{~min}$; 3-甲氧基苯硫酚自偶联副产物二硫醚 12, 260 $\min$.

\section{3 取代苯嗍酸和硫酚反应的一般步骤}

将 1,10 - 菲啰啉 $(0.05 \mathrm{mmol}), \mathrm{Cu}(\mathrm{OAc})_{2} \cdot \mathrm{H}_{2} \mathrm{O}(0.05$ $\mathrm{mmol}) 、 0.75 \mathrm{~mL}$ DMSO 和 $0.75 \mathrm{~mL} \cdot \mathrm{H}_{2} \mathrm{O}$ 分别加入反应 管中, 室温下搅拌 $10 \mathrm{~min}$. 再加入取代苯硼酸 $(0.5$ $\mathrm{mmol})$ 和硫酚 $(0.6 \mathrm{mmol})$, 控温 $60{ }^{\circ} \mathrm{C}$ 搅拌反应 $8 \mathrm{~h}$. 冷却 至室温, 分别加入乙酸乙酯和水各 $3 \mathrm{~mL}$, 分液, 用乙酸 乙酯萃取水相三次 $(3 \mathrm{~mL} \times 3)$, 合并有机相, 用无水 $\mathrm{NaSO}_{4}$ 干燥, 以环己烷为淋洗液用柱层析分离得纯产 品.

对于苯硼酸和 2-颈基苯甲酸的反应，反应结束后， 分别加入 $3 \mathrm{~mL}$ 乙酸乙酯和 $3 \mathrm{~mL} 2 \mathrm{~mol} / \mathrm{L}$ 的 $\mathrm{NaOH}$ 溶液, 充分摚拌后分液, 分离出水相, 用 $1 \mathrm{~mol} / \mathrm{L}$ 的盐酸酸化, 再用二氯甲烷萃取三次, 然后柱层析分离得纯品.

所有产物都为已知化合物, 且通过 ${ }^{1} \mathrm{H}$ NMR 和 ${ }^{13} \mathrm{C}$ NMR 表征, 部分产物的光谱数据如下.

二苯硫醚 ${ }^{[15]}$ : 无色液体; ${ }^{1} \mathrm{H}$ NMR $(300 \mathrm{MHz}$, $\left.\mathrm{CDCl}_{3}\right) \delta: 7.24 \sim 7.39(\mathrm{~m}, 10 \mathrm{H}) ;{ }^{13} \mathrm{C}$ NMR $(75 \mathrm{MHz}$, $\left.\mathrm{CDCl}_{3}\right) \delta: 135.7,131.0,129.2,127.0$; MS (EI) $\mathrm{m} / z$ (\%): $186.4\left(\mathrm{M}^{+}, 100\right), 109.5(6)$.

2-甲苯基苯硫醚 ${ }^{[15]}$ : 无色液体; ${ }^{1} \mathrm{H}$ NMR $(300 \mathrm{MHz}$, $\left.\mathrm{CDCl}_{3}\right) \delta: 7.20 \sim 7.33(\mathrm{~m}, 9 \mathrm{H}), 2.40(\mathrm{~s}, 3 \mathrm{H}) ;{ }^{13} \mathrm{C}$ NMR $(75$ $\left.\mathrm{MHz}, \mathrm{CDCl}_{3}\right) \delta: 140.2,136.5,133.9,133.2,130.9,129.9$, $129.4,128.2,126.9,126.6,20.9$.

2-苯硫基苯甲酸：白色固体；m.p. 166 $168{ }^{\circ} \mathrm{C}$ (lit. ${ }^{[15]}$ m.p. $\left.166.5 \sim 167.0{ }^{\circ} \mathrm{C}\right)$; ${ }^{1} \mathrm{H}$ NMR $(300 \mathrm{MHz}$, $\left.\mathrm{CDCl}_{3}\right) \delta: 13.2($ br s, $1 \mathrm{H}), 7.94(\mathrm{~d}, J=7.5 \mathrm{~Hz}, 1 \mathrm{H}), 7.51 \sim$ $7.55(\mathrm{~m}, 5 \mathrm{H}), 7.36(\mathrm{~d}, J=7.5 \mathrm{~Hz}, 1 \mathrm{H}), 7.23(\mathrm{~d}, J=7.5 \mathrm{~Hz}$, $1 \mathrm{H}), 6.75(\mathrm{~d}, J=7.8 \mathrm{~Hz}, 1 \mathrm{H}) ;{ }^{13} \mathrm{C} \mathrm{NMR}\left(75 \mathrm{MHz}, \mathrm{CDCl}_{3}\right)$ $\delta: 167.5,141.7,135.2,132.5,132.3,131.0,130.2,129.4$, $127.9,127.1,124.8$.

4-甲苯基苯硫醚 ${ }^{44 c]}$ : 无色液体; ${ }^{1} \mathrm{H}$ NMR $(300 \mathrm{MHz}$, $\left.\mathrm{CDCl}_{3}\right) \delta: 7.17 \sim 7.38(\mathrm{~m}, 9 \mathrm{H}), 2.39$ (s, 3H); ${ }^{13} \mathrm{C}$ NMR $(75$ $\left.\mathrm{MHz}, \mathrm{CDCl}_{3}\right) \delta: 137.4,137.0,132.1,131.2,130.0,129.7$, 128.9, 126.3, 21.0; MS (EI) $m / z$ (\%): $200.2\left(\mathrm{M}^{+}, 100\right)$, $185.3(5), 91.5(5)$.

4-溴苯基苯硫醚 ${ }^{[4 c]}$ : 无色液体; ${ }^{1} \mathrm{H}$ NMR $(300 \mathrm{MHz}$, $\left.\mathrm{CDCl}_{3}\right) \delta: 7.24 \sim 7.41(\mathrm{~m}, 7 \mathrm{H}), 7.17(\mathrm{~d}, J=8.4 \mathrm{~Hz}, 2 \mathrm{H}) ;{ }^{13} \mathrm{C}$ NMR $\left(75 \mathrm{MHz}, \mathrm{CDCl}_{3}\right) \delta: 135.5,134.8,132.2,132.0$, 131.5, 129.3, 127.5, 120.8; MS (EI) $m / z(\%): 266.1\left(\mathrm{M}^{+}\right.$, $100), 185.3(50)$

4-异丙苯基苯硫醚 ${ }^{[4 c]}$ : 无色液体; ${ }^{1} \mathrm{H}$ NMR (300 $\left.\mathrm{MHz}, \mathrm{CDCl}_{3}\right) \delta: 7.31 \sim 7.38(\mathrm{~m}, 5 \mathrm{H}), 7.21 \sim 7.28(\mathrm{~m}, 4 \mathrm{H})$, $2.91 \sim 2.99(\mathrm{~m}, 1 \mathrm{H}), 1.29(\mathrm{~d}, J=6.9 \mathrm{~Hz}, 6 \mathrm{H}) ;{ }^{13} \mathrm{C}$ NMR $\left(75 \mathrm{MHz}, \mathrm{CDCl}_{3}\right) \delta: 149.1,137.6,132.7,132.5,130.8$, $129.8,128.1,127.2,34.5,24.6$.

4-甲氧基苯基苯硫醚 ${ }^{[4 \mathrm{c}]}$ : 无色液体; ${ }^{1} \mathrm{H}$ NMR (300 $\left.\mathrm{MHz}, \mathrm{CDCl}_{3}\right) \delta: 7.44(\mathrm{~d}, J=8.7 \mathrm{~Hz}, 2 \mathrm{H}), 7.20 \sim 7.26(\mathrm{~m}$, $5 \mathrm{H}), 6.92(\mathrm{~d}, J=8.7 \mathrm{~Hz}, 2 \mathrm{H}), 3.83(\mathrm{~s}, 3 \mathrm{H}) ;{ }^{13} \mathrm{C}$ NMR $(75$ $\left.\mathrm{MHz}, \mathrm{CDCl}_{3}\right) \delta: 160.6,139.3,136.0,129.6,128.9,126.4$, 125.0, 115.7, 56.0.

\section{2 结果与讨论}

\section{1 优化反应条件}

选用苯硼酸和间甲氧基苯硫酚作为模板底物对反 应条件进行了优化，结果见表 1.

最初选用了 $\mathrm{Cu}_{2} \mathrm{O}-2,2$-联吡啶(bpy)作为催化剂, DMSO 为溶剂, $45{ }^{\circ} \mathrm{C}$ 反应 $8 \mathrm{~h}$, 仅以 $22 \%$ 的收率得到产 品，同时还有 31\%的间甲氧基苯硫酚的自身偶联副产物 二硫醚生成(Entry 1). 如果反应在 $\mathrm{N}_{2}$ 中进行几乎得不到 产品, 但得到 $20 \%$ 的二硫梄(Entry 2). 由于产物和二硫 醚副产物的极性大小相近，二者分离极其困难，所以不 仅要提高产物的收率，而且要减少二硫醚副产物的生 成. 文献[14]中报道苯硼酸参与的反应可以加少量的水, 所以我们在溶剂 DMSO 中加入了 $\mathrm{H}_{2} \mathrm{O}$ [V(DMSO)/ $\left.V\left(\mathrm{H}_{2} \mathrm{O}\right)=2 / 1\right)$. 惊奇地发现，不仅产率明显提高，并且 二硫醚副产物比例明显减少. 水的用量对反应收率有较 大影响, DMSO 与水的体积比为 $1 / 1$ 时得到最高的收率 (Entries 3 5). 在此条件下, 优化了其他的一价铜盐 $\mathrm{CuI}, \mathrm{CuBr}$ 和 $\mathrm{CuCl}$ 以及二价铜盐 $\mathrm{CuO}, \mathrm{CuCl}_{2} \cdot 2 \mathrm{H}_{2} \mathrm{O}$, $\mathrm{CuSO}_{4} \cdot 5 \mathrm{H}_{2} \mathrm{O}$ 和 $\mathrm{Cu}(\mathrm{OAc})_{2} \cdot \mathrm{H}_{2} \mathrm{O}$, 发现除了 $\mathrm{CuSO}_{4} \cdot 5 \mathrm{H}_{2} \mathrm{O}$ 活性稍差外, 其他都能催化反应, 能得到高于 $45 \%$ 的收 
表 1 反应条件的优化 ${ }^{a}$

Table 1 Screening for optimal conditions

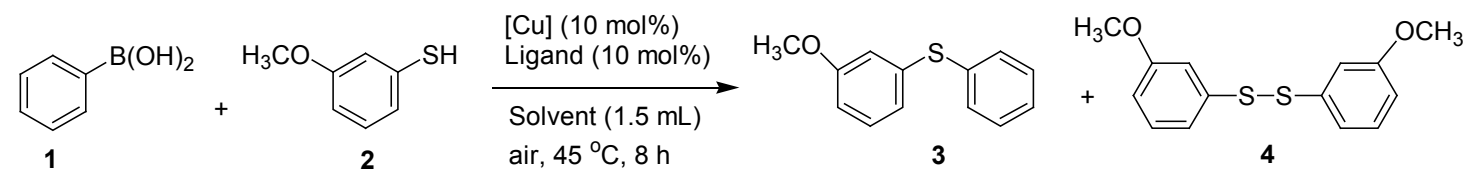

\begin{tabular}{|c|c|c|c|c|c|}
\hline \multirow{2}{*}{ Entry } & \multirow{2}{*}[\mathrm{Cu}]{} & \multirow{2}{*}{ Ligand } & \multirow{2}{*}{ Solvent } & \multicolumn{2}{|c|}{ Yield $^{b} / \%$} \\
\hline & & & & $3^{c}$ & $4^{d}$ \\
\hline 1 & $\mathrm{Cu}_{2} \mathrm{O}$ & bpy & DMSO & 22 & 31 \\
\hline 2 & $\mathrm{Cu}_{2} \mathrm{O}$ & bpy & DMSO & Trace & 20 \\
\hline $3^{e}$ & $\mathrm{Cu}_{2} \mathrm{O}$ & bpy & $\mathrm{DMSO} / \mathrm{H}_{2} \mathrm{O}(V: V=2: 1)$ & 47 & 12 \\
\hline 4 & $\mathrm{Cu}_{2} \mathrm{O}$ & bpy & $\mathrm{DMSO} / \mathrm{H}_{2} \mathrm{O}(V: V=1: 1)$ & 54 & 11 \\
\hline 5 & $\mathrm{Cu}_{2} \mathrm{O}$ & bpy & $\mathrm{DMSO} / \mathrm{H}_{2} \mathrm{O}(V: V=1: 2)$ & 27 & 43 \\
\hline 6 & $\mathrm{CuBr}$ & bpy & $\mathrm{DMSO} / \mathrm{H}_{2} \mathrm{O}(V: V=1: 1)$ & 45 & 13 \\
\hline 7 & $\mathrm{CuI}$ & bpy & $\mathrm{DMSO} / \mathrm{H}_{2} \mathrm{O}(V: V=1: 1)$ & 60 & Trace \\
\hline 8 & $\mathrm{CuCl}$ & bpy & $\mathrm{DMSO} / \mathrm{H}_{2} \mathrm{O}(V: V=1: 1)$ & 47 & 11 \\
\hline 9 & $\mathrm{CuO}$ & bpy & $\mathrm{DMSO} / \mathrm{H}_{2} \mathrm{O}(V: V=1: 1)$ & 61 & 22 \\
\hline 10 & $\mathrm{CuCl}_{2} \cdot 2 \mathrm{H}_{2} \mathrm{O}$ & bpy & $\mathrm{DMSO} / \mathrm{H}_{2} \mathrm{O}(V: V=1: 1)$ & 72 & 11 \\
\hline 11 & $\mathrm{CuSO}_{4} \cdot 5 \mathrm{H}_{2} \mathrm{O}$ & bpy & $\mathrm{DMSO} / \mathrm{H}_{2} \mathrm{O}(V: V=1: 1)$ & 19 & 52 \\
\hline 12 & $\mathrm{Cu}(\mathrm{OAc})_{2} \bullet \mathrm{H}_{2} \mathrm{O}$ & bpy & $\mathrm{DMSO} / \mathrm{H}_{2} \mathrm{O}(V: V=1: 1)$ & 77 & 15 \\
\hline 13 & - & bpy & $\mathrm{DMSO} / \mathrm{H}_{2} \mathrm{O}(V: V=1: 1)$ & ND & 45 \\
\hline 14 & $\mathrm{Cu}(\mathrm{OAc})_{2} \bullet \mathrm{H}_{2} \mathrm{O}$ & - & $\mathrm{DMSO} / \mathrm{H}_{2} \mathrm{O}(V: V=2: 1)$ & Trace & 57 \\
\hline 15 & $\mathrm{Cu}(\mathrm{OAc})_{2} \bullet \mathrm{H}_{2} \mathrm{O}$ & $1,10-\mathrm{Phen} \bullet \mathrm{H}_{2} \mathrm{O}$ & $\mathrm{DMSO} / \mathrm{H}_{2} \mathrm{O}(V: V=1: 1)$ & 81 & 13 \\
\hline 16 & $\mathrm{Cu}(\mathrm{OAc})_{2} \bullet \mathrm{H}_{2} \mathrm{O}$ & $L$-Proline & $\mathrm{DMSO} / \mathrm{H}_{2} \mathrm{O}(V: V=1: 1)$ & 9 & 56 \\
\hline 17 & $\mathrm{Cu}(\mathrm{OAc})_{2} \bullet \mathrm{H}_{2} \mathrm{O}$ & $N, N$-Dimethylethylenediamine & $\mathrm{DMSO} / \mathrm{H}_{2} \mathrm{O}(V: V=1: 1)$ & 21 & 48 \\
\hline 18 & $\mathrm{Cu}(\mathrm{OAc})_{2} \bullet \mathrm{H}_{2} \mathrm{O}$ & $1,10-\mathrm{Phen} \bullet \mathrm{H}_{2} \mathrm{O}$ & $\mathrm{THF} / \mathrm{H}_{2} \mathrm{O}(V: V=1: 1)$ & 13 & 48 \\
\hline 19 & $\mathrm{Cu}(\mathrm{OAc})_{2} \bullet \mathrm{H}_{2} \mathrm{O}$ & 1,10 -Phen $\bullet \mathrm{H}_{2} \mathrm{O}$ & $\mathrm{DMF} / \mathrm{H}_{2} \mathrm{O}(V: V=1: 1)$ & 77 & 11 \\
\hline 20 & $\mathrm{Cu}(\mathrm{OAc})_{2} \bullet \mathrm{H}_{2} \mathrm{O}$ & $1,10-\mathrm{Phen} \bullet \mathrm{H}_{2} \mathrm{O}$ & $\mathrm{EtOH} / \mathrm{H}_{2} \mathrm{O}(V: V=1: 1)$ & 5 & 53 \\
\hline $21^{f}$ & $\mathrm{Cu}(\mathrm{OAc})_{2} \bullet \mathrm{H}_{2} \mathrm{O}$ & $1,10-\mathrm{Phen} \bullet \mathrm{H}_{2} \mathrm{O}$ & $\mathrm{DMSO} / \mathrm{H}_{2} \mathrm{O}(V: V=1: 1)$ & 93 & Trace \\
\hline $22^{f, g}$ & $\mathrm{Cu}(\mathrm{OAc})_{2} \bullet \mathrm{H}_{2} \mathrm{O}$ & $1,10-\mathrm{Phen} \bullet \mathrm{H}_{2} \mathrm{O}$ & $\mathrm{DMSO} / \mathrm{H}_{2} \mathrm{O}(V: V=1: 1)$ & 91 & Trace \\
\hline $23^{f, h}$ & $\mathrm{Cu}(\mathrm{OAc})_{2} \bullet \mathrm{H}_{2} \mathrm{O}$ & $1,10-\mathrm{Phen} \bullet \mathrm{H}_{2} \mathrm{O}$ & $\mathrm{DMSO} / \mathrm{H}_{2} \mathrm{O}(V: V=1: 1)$ & 72 & 9 \\
\hline
\end{tabular}

卓 反应条件: $1(0.5 \mathrm{mmol}), \mathbf{2}(0.6 \mathrm{mmol}),[\mathrm{Cu}](0.05 \mathrm{mmol})$, 配体 $(0.05 \mathrm{mmol})$, 溶剂 $(1.5 \mathrm{~mL})$, 空气下 $45{ }^{\circ} \mathrm{C}$ 反应 $8 \mathrm{~h} ;{ }^{b} \mathrm{GC}-\mathrm{MS}$ 产率 $\left(\right.$ 二苯基二硫醚为内标); ${ }^{c}$ 相 对底物 $1 ;{ }^{d}$ 相对底物 $2 ;{ }^{e}$ 在 $\mathrm{N}_{2}$ 下反应; ${ }^{f} 60{ }^{\circ} \mathrm{C}$ 反应; ${ }^{g}$ 加入 $5 \mathrm{~mol} \% \mathrm{Cu}(\mathrm{OAc})_{2} \bullet \mathrm{H}_{2} \mathrm{O}$ 和 $5 \mathrm{~mol} \% 1,10$-菲啰啉 $\left(1,10-\mathrm{phen} \cdot \mathrm{H}_{2} \mathrm{O}\right) ;{ }^{h}$ 加入 $2 \mathrm{~mol} \% \mathrm{Cu}(\mathrm{OAc})_{2} \cdot \mathrm{H}_{2} \mathrm{O}$ 和 $2 \mathrm{~mol} \%$ 1,10-菲啰啉.

率(Entries 6 12), 其中 $\mathrm{Cu}(\mathrm{OAc})_{2} \bullet \mathrm{H}_{2} \mathrm{O}$ 的催化活性最 高, 能得到 77\%收率, 但是二硫醚的副产物比例依然较 大. 反应体系中铜和配体是必需的, 二者缺一都得不到 产物, 但是可以得到 $45 \%$ 以上的二硫醚副产物(Entries 13, 14). 接着将常见的配体如 1,10-菲啰啉、 $L$-脯胺酸和 $\mathrm{N}, \mathrm{N}$-二甲基乙二胺等用于催化体系中, 发现 1,10-菲啰 啉能得到最好 $81 \%$ 的收率(Entries 15 17). 同时也考察 溶剂的影响, 将 DMSO 用其他水溶性的溶剂 THF, DMF 或 EtOH 代替, 不但收率降低, 而且二硫醚副产物比例 也增加(Entries 18 20), 当反应温度升高到 $60{ }^{\circ} \mathrm{C}$ 时, 收 率提高到 $93 \%$, 二硫醚副产物基本消失(Entry 21). 在 60 ${ }^{\circ} \mathrm{C}$ 反应，当催化剂 $\mathrm{Cu}(\mathrm{OAc})_{2} \cdot \mathrm{H}_{2} \mathrm{O}-1,10-\mathrm{phen} \cdot \mathrm{H}_{2} \mathrm{O}$ 的用 量降低到 $5 \mathrm{~mol} \%$, 收率没有明显的变化, 但是当催化剂 用量继续降低到 $2 \mathrm{~mol} \%$ 时, 收率降低到 72\% (Entries $22,23)$. 所以优化后的反应条件选择: $\mathrm{Cu}(\mathrm{OAc})_{2} \cdot \mathrm{H}_{2} \mathrm{O}-$ 1,10 -菲啰啉 (5 mol\%)为催化剂, $\mathrm{DMSO} / \mathrm{H}_{2} \mathrm{O}(V: V=1$ : 1)为溶剂, 反应温度为 $60{ }^{\circ} \mathrm{C}$.

\section{2 硫酚和芳基硼酸的反应}

在优化的反应条件下, 考察了取代苯硫酚和芳基硼 酸的反应, 结果见表 2.

从表 2 中可知, 各种取代苯硼酸和硫酚都能顺利地 反应，以中等到高的收率得到产物. 从苯硼酸与各种取 代苯硫酚的反应可以看出，苯硫酚上取代基的空间效应 对反应有影响. 相同取代基邻位取代比对位取代活性要 低, 如 $\mathrm{Me}, \mathrm{Cl}$ 和 $\mathrm{Br}$ 取代邻位时，收率分别为 $50 \%, 73 \%$ 和 79\%, 但是当这些基团取代到对位时, 收率分别增加 到 $71 \%, 78 \%$ 和 $87 \%$ (Entries 3 6 vs. 8 10). 当位阻更 大的 2-䒬酚与苯硼酸反应时, 仅仅得到 $54 \%$ 的收率

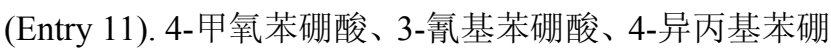
酸和苯硫酚反应，能以 $72 \% \sim 86 \%$ 的收率得到相应的硫 醚(Entries 12 14). 反应体系中能允许取代基 $\mathrm{F}, \mathrm{Cl}, \mathrm{Br}$, $\mathrm{COOH}$ 和 $\mathrm{CN}$ 的存在, 具有较好的普适性. 含杂环的 2颈基噻吩与苯硼酸反应以 $69 \%$ 的收率得到产物(Entry 16). 但是脂肪硫醇, 如芐基硫醇和正丁基硫醇与硼酸 
表 2 取代苯硫酚和取代苯嗍酸的反应 ${ }^{a}$

Table 2 Reaction of arylboronic acids with thiols
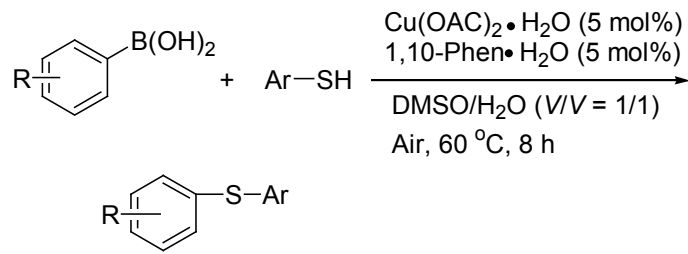

\begin{tabular}{cllc}
\hline Entry & $\mathrm{R}$ & \multicolumn{1}{c}{ Ar } & Yield $^{b} / \%$ \\
\hline 1 & $\mathrm{H}$ & 3-Methoxyphenyl & 88 \\
2 & $\mathrm{H}$ & Phenyl & 93 \\
3 & $\mathrm{H}$ & 2-Tolyl & 50 \\
4 & $\mathrm{H}$ & 2-Chlorophenyl & 73 \\
5 & $\mathrm{H}$ & 2-Bromophenyl & 79 \\
6 & $\mathrm{H}$ & 2-Fluorophenyl & 86 \\
7 & $\mathrm{H}$ & 2-Carboxyphenyl & 63 \\
8 & $\mathrm{H}$ & 4-Tolyl & 71 \\
9 & $\mathrm{H}$ & 4-Chlorophenyl & 78 \\
10 & $\mathrm{H}$ & 4-Bromophenyl & 87 \\
11 & $\mathrm{H}$ & 2-Naphthyl & 54 \\
12 & $4-\mathrm{Me}$ & Phenyl & 85 \\
13 & $3-\mathrm{CN}$ & Phenyl & 72 \\
14 & $4-i$-Pr & Phenyl & 82 \\
15 & $4-\mathrm{OMe}$ & Phenyl & 86 \\
16 & $\mathrm{H}$ & 2-Thienyl & 69 \\
18 & $\mathrm{H}$ & Benzyl & 28 \\
17 & $\mathrm{H}$ & n-Butyl & $<5$ \\
\hline
\end{tabular}

${ }^{a}$ 反应条件: 芳基硼酸 $(0.5 \mathrm{mmol})$, 硫酚 $(0.6 \mathrm{mmol}), \mathrm{Cu}(\mathrm{OAc})_{2} \cdot \mathrm{H}_{2} \mathrm{O}(5$ $\mathrm{mol} \%), 1,10$-菲啰啉 (5 mol\%), DMSO/ $\mathrm{H}_{2} \mathrm{O}(V: V=1: 1)(1.5 \mathrm{~mL})$, 空气下 $60{ }^{\circ} \mathrm{C}$ 反应 $8 \mathrm{~h} ;{ }^{b}$ 分离收率.

反应，收率不理想(Entries 17, 18).

\section{3 机理探讨}

芳基硼酸与含硫化合物反应合成硫醚的机理已有 报道 ${ }^{[13,14 a, 15]}$. 它可能有两个途径(图 1), 首先水与 $\mathrm{ArB}(\mathrm{OH})_{2}$ 反应生成活性更高的 $\mathrm{PhB}(\mathrm{OH})_{3}^{-}{ }^{[17]}$, 再与 $\mathrm{Cu}$ 的配合物 $\mathrm{A}$ 反应生成 $\mathbf{B}$, 然后一种可能 $\mathbf{B}$ 与硫酚反应生 成 $\mathbf{D}$, 再还原消去给出产物, 同时生成 $\mathbf{E}$, 氧化后回到 A，完成催化循环. 另一种可能是硫酚氧化生成二硫醚, 然后与 B 反应, 给出产物, 同时生成低活性的中间体 $\mathbf{C}^{[13]}, \mathbf{C}$ 与活性中间体 $\mathrm{ArB}(\mathrm{OH})_{3}^{-}$反应生成 $\mathbf{D}$, 然后同第 一途径完成余下的催化循环. 已有多例芳基硼酸参与的 反应中加水, 同时水能促进反应的报道 ${ }^{[18]}$. 水能促进这 类反应，一方面可能水能增加 $\mathrm{ArB}(\mathrm{OH})_{2}$ 的溶解，另一 方面水能与 $\mathrm{ArB}(\mathrm{OH})_{2}$ 反应生产活性更高的 $\mathrm{ArB}(\mathrm{OH})_{3}^{-}$, 从而与低活性中间体 $\mathbf{C}$ 反应成为可能 ${ }^{[17,19]}$.

为了研究第二种机理是否存在, 我们将二(3-甲氧 基苯)硫醚与苯硼酸在优化的条件下反应，以 $55 \%$ 的收 率得到了产物(Eq. 2). 在前述优化反应条件，也生成了 大量的硫酚自偶联产物二硫醚, 这些都表明第二种机理

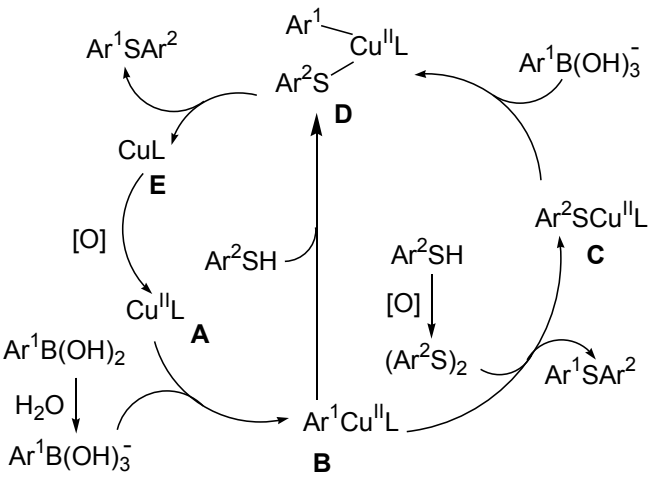

图 1 可能的反应机理

Figure 1 Proposed reaction mechanism

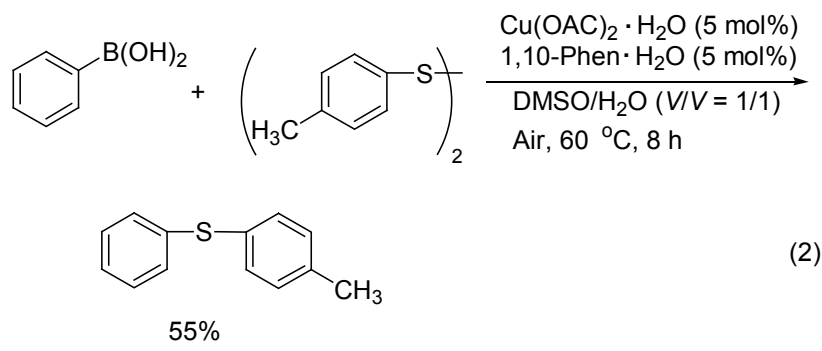

是可能存在的.

\section{3 结论}

本文以廉价的醋酸铜-1,10-菲罗啉为催化剂, 催化 芳基硼酸和硫酚的偶联反应，以较高的收率合成系列的 非对称的硫醚，二硫的副产物极少，易于分离. 相比传 统上用芳基卤为芳化试剂制备硫醚，体系中不需要高温 和强碱，所用的铜盐廉价无毒易得. 相比 Feng 小组 ${ }^{[15]}$ 的研究, 尽管反应温度偏高, 但是直接在空气中进行, 不需要加大量的 $n-\mathrm{Bu}_{4} \mathrm{NOH}$ 作为碱, 且部分产物的收率 也有所提高. 水明显地促进反应的进行，详细的催化机 理研究正在进行中.

\section{References}

[1] (a) Neilsen, S. F.; Neilsen, E. O.; Olsen, G. M.; Liljefors, T.; Peters, D. J. Med. Chem. 2000, 43, 2217.

(b) Navarro, R.; Perrino, M. P.; Tardajos, M. G.; Reinecke, H. Macromolecules 2010, 43, 2377.

[2] Guilarte, V.; Fernández-Rodríguez, M. A.; García-García, P.; Hernando, E.; Sanz, R. Org. Lett. 2011, 13, 5100.

[3] Taniguchi, N. J. Org. Chem. 2004, 69, 6904.

[4] (a) Kwong, F. Y.; Buchwald, S. L. Org. Lett. 2002, 4, 3517.

(b) Lv, X.; Bao, W.-L. J. Org. Chem.2007, 72, 3863.

(c) Sperotto, E.; Klink, G. P. M.; Vries, J. G.; Koten, G. J. Org. Chem. 2008, 73, 5625.

[5] (a) Yudin, A. K. Catalyzed Carbon-Heteroatom Bond Formation, Wiley-VCH, Weinheim, 2010, pp. 69 113.

(b) Bichler, P.; Love, J. A. Top. Organomet. Chem. 2010, 31, 39.

(c) Eichman, C. C.; Stambuli, J. P. Molecules 2011, 16, 590.

[6] Jiang, Y.; Qin, Y.; Xie, S.; Zhang, X.; Dong, J.; Ma, D. Org. Lett. 
2009, 11,5250

[7] Ke, F.; Qu, Y.; Jiang, Z.; Li, Z.; Wu, D.; Zhou, X. Org. Lett. 2011, 13,545 .

[8] (a) Reddy, K. H. V.; Reddy, V. P.; Shankar, J.; Madhav, B.; Kumar, B. S. P. A.; Nageswar, Y. V. D. Tetrahedron Lett. 2011, 52, 2679.

(b) Firouzabadi, H.; Iranpoor, N.; Gholinejad, M. Adv. Synth. Catal. 2010, 119.

[9] Jiang, Z.; She, J.; Lin, X.-F. Adv. Synth. Catal. 2009, 2558.

[10] Kumar, S.; Engman, L. J. Org. Chem. 2006, 71, 5400.

[11] Herradura, P. S.; Pendola, K. A.; Guy, R. K. Org. Lett. 2000, 2, 2019.

[12] Jammi, S.; Sakthivel, S.; Rout, L.; Mukherje, T.; Mandal, S.; Mitra, R.; Sha, P.; Punniyamurthy, T. J. Org. Chem. 2009, 74, 1971.

[13] Savarin, C.; Srogl, J.; Liebeskind, L. S. Org. Lett. 2002, 4, 4309.

[14] (a) Taniguchi, N. J. Org. Chem. 2007, 72, 1241.

(b) Taniguchi, N. Synlett 2006, 1351.

(c) Luo, P.-S.; Wang, F.; Li, J.-H.; Tang, R.-Y.; Zhong, P. Synthesis 2009, 921.

[15] Xu, H.; Zhao, Y.; Feng, T.; Feng,Y. J. Org. Chem. 2012, 77, 2878.

[16] (a) Yang, M.; Zhu, C.; Yuan, F.; Huang, Y.; Pan, Y. Org. Lett.
2005, 7, 1927.

(b) Yang, M.; Liu, F. J. Org. Chem. 2007, 72, 8969.

(c) Yang, M.; Yan, G.; Zheng, Y. Tetrahedron Lett. 2008, 49, 6471.

(d) Yang, M.; Pei, J.; Yan, G.; Zheng, Y. Lett. Org. Chem. 2011, 8 , 587.

[17] Hall, D. G.; Suzuki, A. In Boronic Acids, Vol. 2, Ed.: Hall, D. G., Wiley-VCH Verlag, Weinheim, 2011, p. 9 .

[18] In metal-catalyzed organic reaction using organoboronic acid, water has been added: (a) Miyaura, N.; Suzuki, A. Chem. Rev. 1995, 95, 2457.

(b) Hayashi, T.; Yamasaki, K. Chem. Rev. 2003, 103, 2829.

Water can improve metal-catalyzed organic reaction using organoboron compounds, see: (c) Guan, B.-T; Wang, Y.; Li, B.-J.; Yu, D.-G.; Shi, Z. -J. J. Am. Chem. Soc. 2008, 130, 14468. And also please see Ref. [14a].

[19] (a) Liu, L.-F.; Zhang, Y.-H.; Wang, Y. G. J. Org. Chem. 2005, 70, 6122.

(b) Xin, B.-W. Ph.D. Dissertation, Zhejiang University, Hangzhou, 2010, p. 18 (in Chinese).

(辛炳炜, 博士论文, 浙江大学, 杭州, 2010, p. 18.) 